\title{
Properties of star forming regions in the Magellanic Clouds
}

\author{
Mónica Rubio \\ Departamento de Astronomía, Universidad de Chile, Casilla 36-D, Santiago, Chile \\ email: mrubio@das.uchile.cl
}

\begin{abstract}
Understanding the process of star formation in low metallicity systems is one of the key studies in the early stages of galaxy evolution. The Magellanic Clouds, being the nearest examples of low metallicity systems, allow us to study in detail their star forming regions. As a consequence of their proximity we can resolve the molecular clouds and the regions of star formation individually. Therefore we can increase our knowledge of the interaction of young luminous stars with their environment. We will present results of multiwavelenghts studies of LMC and SMC massive star forming regions, which includes properties of the cold molecular gas, the embedded young population associated with molecular clouds, and the interaction of newly born stars with the surrounding interstellar medium, based on ASTE and APEX submillimeter observations complemented high sensitivity NIR groud based observations and Spitzer results.
\end{abstract}

Keywords. stars: formation, ISM: clouds, H II regions, Magellanic Clouds

\section{Introduction}

The molecular clouds constitute the cold interstellar material where stars are born. The rate of star formation depends on the quantity of molecular gas and on the concentration in the clouds as a result of gravitational bounding. The characterization of the properties of these clouds and of the star formation efficiency is a key factor to understand the evolution of galaxies.

The Small and Large Magellanic Clouds (SMC and LMC) are the two closest galaxies to the Sun that are rich in gas. These are two irregular galaxies where the structure of interstellar matter is largely influenced by the action of new stars on their environment rather than by the large scale dynamics of high mass spiral galaxies such as the Milky Way. The two clouds share another important characteristic of galaxies in the early stage of evolution. The presence of heavy elements (metallicity) is 10 to 4 times weaker than that of the Solar neighbourhood. The Magellanic Clouds are therefore considered as the nearest environments that allow to study interstellar matter, gas and dust, in the early stages of galaxy evolution.

As a consequence of their proximity, the observations resolve the molecular clouds and the regions of star formation individually. Nevertheless, it is difficult to trace the cold gas because $\mathrm{H}_{2}$ is not directly observable in these conditions. It is necessary to employ tracers. The Magellanic Clouds have been extensively observed in the first rotation transitions of the $\mathrm{CO}$ molecule, but these observations leave many uncertainties on the mass and structure of the clouds. The photo-chemical models suggest that a dominant part of the molecular gas in low metallicity galaxies is weak in CO.

Multiple molecular clouds in the Magellanic Clouds have been surveyed based on CO line emission. These observations show that the CO emission in the Magellanic Clouds is very weak. In effect, the $\mathrm{CO}$ emission compared with star formation tracers, such as the band $\mathrm{B}$ luminosity or the $\mathrm{H} \alpha$ luminosity, is much weaker than in normal galaxies (Leroy 
et al. 2007). Even more, the comparison of the dynamic masses of molecular gas and of the mass derived from CO luminosity gives very disperse values of the conversion factor $X_{\mathrm{CO}}=N\left(\mathrm{H}_{2}\right) / I_{\mathrm{CO}}$, and in average 10 times greater than in our galaxy (Rubio et al. 1993; Mizuno et al. 2001).

\section{Properties of molecular clouds}

Giant Molecular Clouds (GMCs) properties have been derived from complete CO surveys in the Magellanic Clouds. The NANTEN survey covered the LMC (Fukui et al. 1999, 2008) and SMC (Mizuno et al. 2001) at 2.4' resolution, surpassing the $8.8^{\prime}$ resolution survey (Cohen et al. 1988; Rubio et al. 1991). The NANTEN survey has derived the properties of GMCs of typical sizes of about $50 \mathrm{pc}$ and a summary of these properties is given by Mizuno, in these proceedings.

Higher angular resolution observations were possible with the $15 \mathrm{~m}$ SEST telescope but these did not cover the entire galaxies. The ESO-SEST Key Programme: "CO in the Magellanic Clouds" is the best available high angular $\mathrm{CO}(1-0)$ survey with 20 pc spatial resolution of the Magellanic Clouds. In a series of articles, the properties of the surveyed molecular clouds were given. These can be summarized as follows: The LMC has molecular clouds with sizes between 10 and $40 \mathrm{pc}$, line widths between 2.5 and $10 \mathrm{~km}$ $\mathrm{s}^{-1}$ and CO luminosities between $0.8 \times 10^{3}$ and $1.3 \times 10^{4}$. The SMC molecular clouds have sizes between 10 and $20 \mathrm{pc}$, line widths between 1.6 and $6.4 \mathrm{~km} \mathrm{~s}^{-1}$ and CO luminosities between $0.5 \times 10^{3}$ to $6.4 \times 10^{4}$.

In general, these molecular clouds at SEST resolution follow a linewidth-size relationship where $\Delta v \propto R^{0.5}$ (Israel et al. 1993; Rubio et al. 1993, etc.), typical of gravitationally bound molecular clouds as found in our Galaxy and described by Larson (1981).

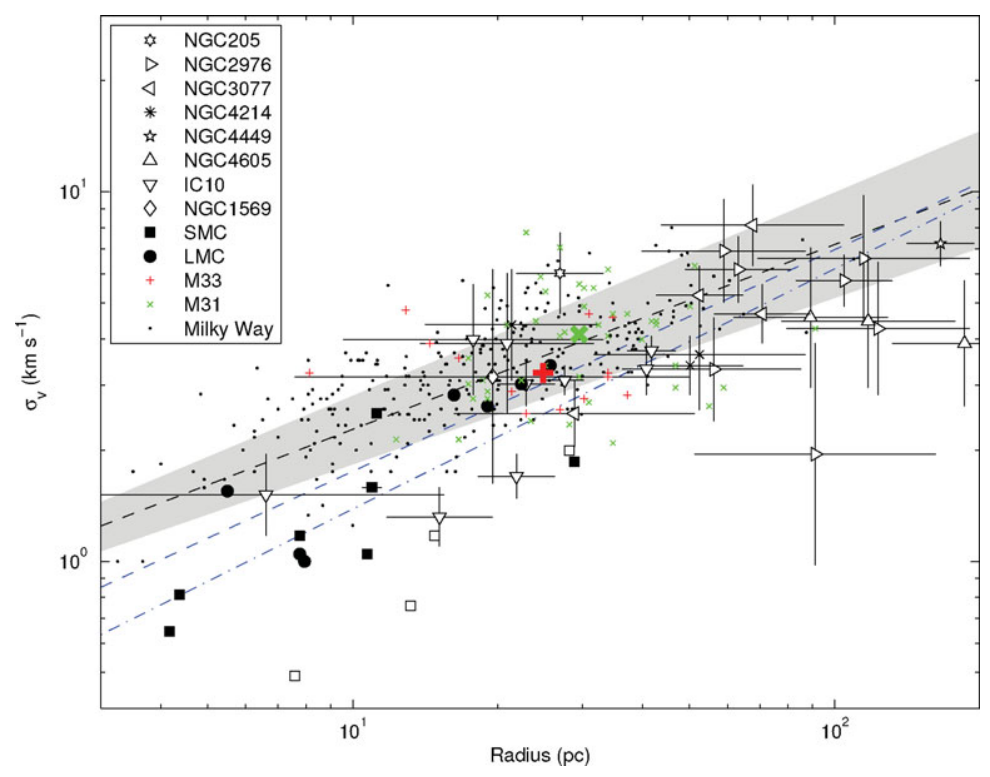

Figure 1. Extragalactic luminosity line width relation for molecular clouds from Bolatto et al. (2008, their figure 2). Measurements for different galaxies are indicated with open and filled symbols depending on whether they were obtained from $\mathrm{CO}(1-0)$ or $\mathrm{CO}(2-1)$ observations, respectively. The grey bar is the relation for Galactic GMCs (Solomon et al. 1987) and the blue dotted line the one obtained considering only the dwarf galaxies. 
A mayor difference found in the properties of Magellanic molecular clouds and Galactic molecular clouds is that Magellanic GMCs show CO luminosities a factor of 5 to 10 weaker than those found in our Galaxy for similar linewidths. This result is still valid for CO clouds observed at $20 \mathrm{pc}$ resolution but the factor decreases to 3 to 5 times less luminous for the LMC and SMC, respectively. This difference has been an important result in revealing that the $\mathrm{CO}$ emission may not be tracing the total molecular mass in low metallicity molecular clouds. Thus, it has become essential for the study of star formation in these galaxies to determine the total molecular gas mass of these clouds.

\subsection{Molecular mass}

To determine the amount of molecular gas is difficult as the cold $\mathrm{H}_{2}$ gas is almost impossible to observe. The most used method to derive the mass in molecular gas is through the CO luminosity of the molecular cloud. In our Galaxy, an empirical relationship has been determined between the column density of $\mathrm{H}_{2}$ and the $\mathrm{CO}$ velocity-integrated emission of the CO cloud. This relationship, calibrated by gamma-ray measurements, virial mass comparison, and extinction measurements (Bloemen et al. 1986) gives consistent results and a calibration factor, known as the $X$ factor, allows to deduce the cloud molecular mass from its $\mathrm{CO}$ emission.

In the Magellanic Clouds, all the CO observations have consistently given a lower molecular mass of the clouds when determined by their CO luminosity and compared to virial mass determination using their $\mathrm{CO}$ linewidths and size. This result is also found even when we have had a ten-fold spatial resolution improvement in the observations as achieved by SEST CO(2-1) observations.

The explanation of this result has been argued as follows: the CO molecule in the Magellanic Clouds, and hence in low metallicty systems, is photodissociated and only traces part of the molecular cloud, keeping large envelopes of $\mathrm{H}_{2}$ hidden from the observations. The CO molecule survives only when it is shielded from the UV radiation field. In the LMC and SMC, this shielding occurs at column densities of molecular $\mathrm{H}_{2}$ gas about 0.1 times the column densities of molecular clouds in our Galaxy of $N\left(\mathrm{H}_{2}\right) \approx 1-1.5 \times 10^{21}$ $\mathrm{cm}^{-2}$. The molecular hydrogen molecule self-shields at a column density lower than CO, the UV photon penetration is much larger, and CO only traces the dense core. As a consequence the molecular mass in molecular clouds in low metallicity systems is mainly $\mathrm{H}_{2}$ and not $\mathrm{CO}$.

Bolatto et al. (2008) have made a comparison of molecular cloud properties in extragalactic systems, including the LMC and SMC, and found that extragalactic GMCs follow approximately similar size-linewidth, luminosity-size, and luminosity-linewidth relations as Galactic molecular clouds. But the SMC molecular cloud properties deviate from typical Galactic molecular cloud properties much more than LMC molecular clouds (Figure 1). The SMC shows lower CO luminosity for similar size as Galactic clouds but smaller linewidths. Thus, these clouds may not be supported gravitationally and other mechanisms might be playing a role, e.g., magnetic fields and/or turbulence. The LMC with only a $1 / 4 \mathrm{Z}_{\odot}$ metallicity does not show this deviation. The impact of the metallicity on these results is still to be explored as the range of metallicities of his sample is in average $1 / 5$ that of the Milky Way. Observationally this is a difficult problem as low metallicity systems have very weak $\mathrm{CO}$ emission and thus require large amounts of telescope time to detect its emission. The SMC, due to its proximity, is therefore an excellent nearby system to study and determine the molecular cloud properties in low metallicity systems.

An alternative method to determine the molecular mass of molecular clouds is by their dust emission. The dust emission peaks in the submillimeter spectral range and the 

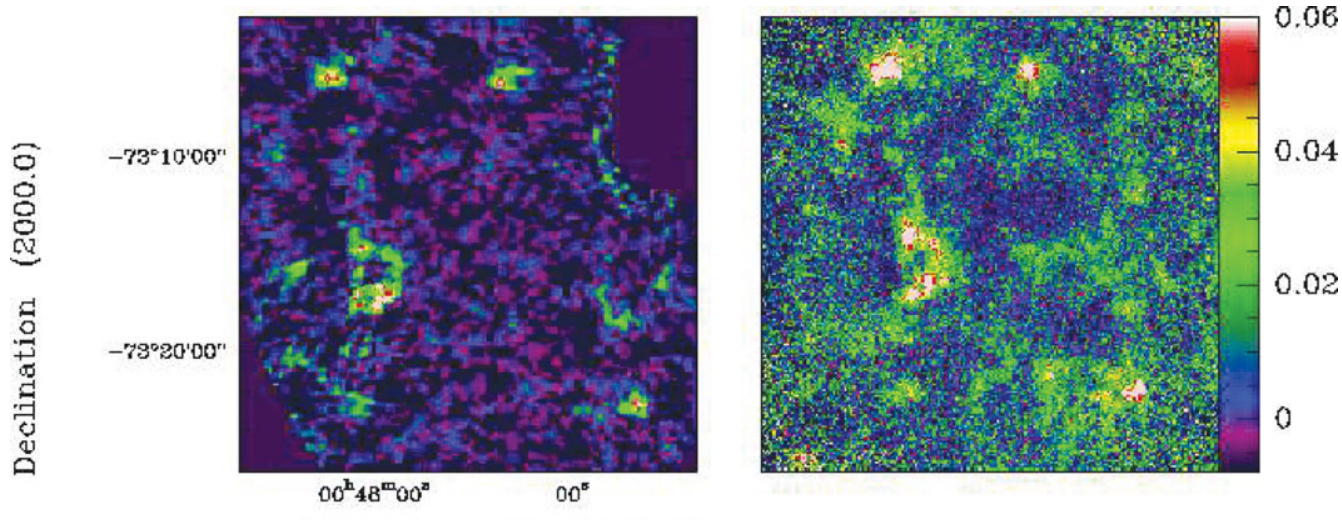

Right Ascension (2000.0)

Figure 2. The continuum emission of the SW region of the SMC, with in the left panel the 1.2-mm SIMBA image and in the right one the $850 \mu \mathrm{m}$ LABOCA image. The bright sources, observed also in $\mathrm{CO}$, are seen at both frequencies while at $850 \mu \mathrm{m}$ extended emission is clearly seen in the region. The scale is $\mathrm{mJy}_{\mathrm{beam}}{ }^{-1}$.

installation of mm and submm bolometer cameras in Chile provide a unique opportunity to measure the continuum dust emission from LMC and SMC molecular clouds. These observations can be critical to resolve the discrepancy between the virial mass and the CO mass determination.

The first 1.2-mm continuum observations of molecular clouds in the Magellanic Clouds were done with the SIMBA bolometer at the SEST telescope in La Silla, Chile. In the SMC, Rubio et al. (2004) reported the results for a cold quiescent cloud in which they found that the gas mass derived from the continuum dust emission was larger than the virial mass determination of the cloud. This result was also found in studies of several molecular clouds in the SW region of the SMC Bar by Bot et al. (2007) as well as in the active star forming region N 66 (Perez 2006). The analysis of observations of the SMC has shown for many clouds in the same region that the masses of gas deduced from the dust emission were ten times higher than those deduced by the application of the virial theorem using the size of the clouds and the gas velocity dispersion measured in CO (Rubio et al. 2004; Bot et al. 2007; fig. 1 of Bot et al., these proceedings). This large difference in mass is surprising. It is larger than the uncertainty on the emissivity of grains and of the gas-to-dust mass ratio. It is known that for weak metallicity the CO molecule does not trace but the densest regions of molecular clouds (Lequeux et al. 1994). It is possible that the dynamics of these regions does not trace the gravitational potential of the cloud. Bot et al. (2007) have proposed that a significant part of the support of molecular clouds against gravitation could be due to a magnetic field. The importance of the magnetic field depends on the mechanism of cloud formation. Its role must be more important where the clouds are created by unidirectional compression on the surfaces of the bubbles blown by star clusters rather than by the isotropic action of gravity.

New observations done at $0.8 \mu \mathrm{m}(350 \mathrm{GHz})$ using the LABOCA bolometer at the $A P E X$ telescope confirm that in the SW region of the SMC the molecular mass derived from the dust emission is larger than the virial mass derived from $\mathrm{CO}(2-1)$ observations (Bot et al., these proceedings). Figure 2 shows the 1.2-mm SIMBA and the 0.8-mm LABOCA images of the SW SMC Bar. The bright sources have been mapped in CO. The $0.8 \mu \mathrm{m}$ LABOCA image shows diffuse continuum emission almost all over the observed area. Bot et al. (these proceedings) have found that after subtracting the free-free 
emission and CO line contribution, and using a range of possible dust emissivities, analyzing different temperature dependencies, and using the gas-to-dust ratios as determined for the SMC, the gas mass derived from the dust emission continues to be consistently larger than the virial mass determination, being a factor of 2 to 10 larger. Analysis is under way but the confirmation that dust emission gives larger gas masses is consistent with the fact that SMC molecular clouds must have large envelopes of $\mathrm{H}_{2}$.

These studies are based on a certain number of hypothesis on the dust properties in the millimeter domain. Little is known about dust outside the Galaxy, particularly the impact of metallicity on its composition and the ratio in gas mass over dust (Reach et al. 2000; Weingartner \& Draine 2001; Bot et al. 2004). Some studies of dust emission in low metallicity galaxies farther than the Magellanic Clouds have shown variations in dust properties (Galliano et al. 2003, 2005) but the molecular clouds in these galaxies are not resolved and the variations are not currently well understood. In the Galaxy, variations of dust millimeter emissivity have been observed between cold molecular clouds, regions of hot-star formation and the diffuse medium (Dupac et al. 2003). These variations have been associated with the evolution in the composition and the distribution in size of dust by the formation of mantles and grain coagulation (see Boulanger, these proceedings).

There have been indirect methods to determine the amount of molecular mass in the molecular clouds by using the mid-IR emission from gas and dust.

In the SMC, Leroy et al. (2007), using the $160 \mu \mathrm{m}$ Spitzer images (Bolatto et al. 2007) plus the H I gas emission determined molecular hydrogen columnn densities which they compared to the molecular clouds as mapped by the NANTEN $\mathrm{CO}(1-0)$ survey. They obtain that the $160 \mu \mathrm{m}$ emission has a spatial extension larger than the volume where CO emission has been detected, consistent with molecular clouds having a large envelope of $\mathrm{H}_{2}$ molecular gas and dense but smaller $\mathrm{CO}$ cores. The mid-IR images show large dusty envelopes suggesting that low metallicity dense clouds have low-density and CO-free $\mathrm{H}_{2}$ envelopes. A large fraction of the molecular mass could be contained in such lower density envelopes (Lequeux et al. 1994).

This result is also confirmed by a recent a study of one SMC molecular cloud (N 83) (Leroy et al., in preparation) in which they have combined the $160 \mu \mathrm{m}$ and $70 \mu \mathrm{m}$ Spitzer data, the H I emission, and the high resolution SEST CO (2-1) data with 10 parsec resolution. Leroy and collaborators derive from the $\mathrm{HI}$ and $160 \mu \mathrm{m}$ opacities a gas-to-dust ratio 14 times larger than that of the diffuse gas in our Galaxy. They find that the $\mathrm{H}_{2}$ densities of molecular gas in this cloud are larger than $350 \mathrm{M}_{\odot} \mathrm{pc}^{-2}$ while in the molecular ring in the Milky Way $N\left(\mathrm{H}_{2}\right) \sim 8 \mathrm{M}_{\odot} \mathrm{pc}^{-2}$. The CO emission comes from $A_{\mathrm{V}} \sim 1.6 \mathrm{mag}$, consistent with the PDR models of SMC molecular clouds where $\mathrm{CO}$ selfshields much deeper than the molecular hydrogen as modeled by Lequeux et al. (1994) using SMC gas conditions, i.e., a 1/10 lower dust content, a lower abundance of $\mathrm{C}$ and $\mathrm{O}$, and a higher, about 1000 larger UV radiation field, than the ISM conditions of the Galaxy. Leroy et al. (these proceedings) suggest that the sizes of the dense molecular clumps are 1/10 of those of the dense clumps in Galactic molecular clouds. The small size of the dense clumps would explain that the conversion factor in the SMC would be larger than that determined in the Galaxy implying that the mass determination from CO luminosities has to be lower than in Galacic molecular clouds. For example, the typical mass of a molecular cloud in the SMC is $M=10^{4} \mathrm{M}_{\odot}$ while in the Galaxy a low mass star forming region such as $\rho$ Ophiuchus has a mass of $M \sim 5 \times 10^{5} \mathrm{M}_{\odot}$ for the principal CO clump. Thus, star formation can be quite different in these low metallicity molecular clouds. 


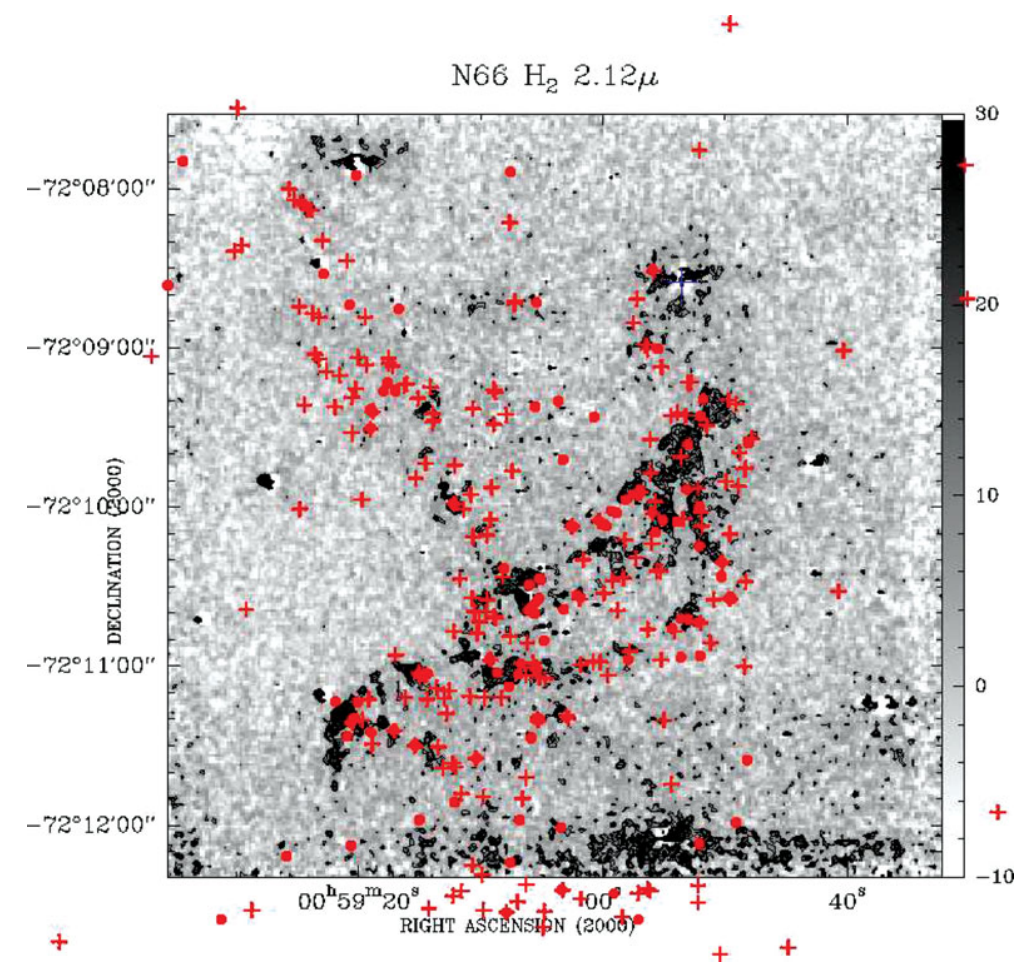

Figure 3. The $2.12 \mu \mathrm{m} \mathrm{H}_{2}$ image of N 66. The Spitzer YSO list of Simon et al. (2007) is plotted with crosses and the near-IR sources with $J-K_{\mathrm{s}}>1 \mathrm{mag}$ as filled circles. Young massive objects follow the gas emission and concentrate on the $\mathrm{H}_{2}$ knots.

\subsection{Star formation}

Stellar associations in the Magellanic Clouds contain the richest sample of young bright stars and thus our knowledge of their young massive stars has been collected from studies of such stellar systems (e.g., Massey et al. 2002). Almost every young association coincides with one or more H II regions as catalogued by Henize (1956) and Davies et al. (1976). These young associations also host large numbers of PMS stars (Nota et al. 2006; Gouliermis et al. 2006). These young OB associations correlate with the distribution of molecular clouds. In the LMC, young associations with ages less than one million years are found associated with GMCs (Yamaguchi et al. 2001; Fukui et al. 2008).

Observationally the study of star formation in the Magellanic Clouds can be adressed combining ground-based observations with IR capabilities of $8 \mathrm{~m}$-class telescopes operating in the southern hemisphere (Chile), and space-based NICMOS/HST and Spitzer observations. Several studies have investigated massive star forming regions and their molecular clouds.

In the LMC, these studies include the bright Giant H II region 30 Doradus and its massive super star cluster R 136 (Rubio et al. 1998; Walborn et al. 1999; Brandner et al. 2001; Oliveira et al. 2006), the second largest H II region N 11 (Barbá et al. 2003), the young H II region N 159 (Jones et al. 2005; Rubio et al. 2008), and a compact H II region N 4 (Contursi et al. 2007).

In the SMC, these studies have concentrated on the brightest $\mathrm{H}$ II region $\mathrm{N} 66$ and its central cluster NGC 346 (Contursi et al. 2000; Rubio et al. 2000), the H II regions N 27 (LIRS 49) and N 12 (LIRS 36), and NGC 602/N 90 (Gouliermis et al. 2008). 
Deep near-IR $J H K$ imaging has been obtained towards these regions to study the embedded population in molecular clouds. Infrared color-color and color-magnitude diagrams have shown the existence of infrared sources that have colors consistent with massive YSOs. Their colors imply important IR excess and/or large extinction.

In N 66 in the SMC, Nota et al. (2006) and Sabbi et al. (2008), using HST observations. determined the spatial distribution of PMS stars and found a very good correlation of PMS and young stars with CO emission peaks. A comparison of their catalogue and the near-IR photometry of ISAAC/VLT images of IR sources with $\left(K_{\mathrm{s}}-H\right)>1.5 \mathrm{mag}$ shows a strong association of the PMS star location to the $\mathrm{H}_{2} 2.12 \mu \mathrm{m}$ gas emission with a large concentration towards molecular knots in N 66 (Rubio et al. 2000; Rubio 2007).

Using the available Spitzer LMC (Meixner et al. 2006) and SMC (Bolatto et al. 2007) mid IR imaging, complete point source catalogues have been made and used to select embedded and/or young stellar objects based on their IRAC color diagrams. In the SMC, Simon et al. (2007) produced a point source catalogue and determined YSO candidates from SED fits. These YSO candidates show a very strong spatial correlation with the molecular cloud peaks and $\mathrm{H}_{2} 2.12 \mu \mathrm{m}$ dense knots as seen in Figure 3. The $\mathrm{H}_{2}$ dense knots are most probably PDRs formed by the embedded massive stars which are photodissociating their natal cloud. IR spectroscopy of three IR embedded sources associated with $\mathrm{H}_{2}$ knots in N 66 confirm their massive YSO's nature (Rubio \& Barbá 2008). Similarly, NGC 602/N 90 hosts several Spitzer YSO candidates associated with this massive star forming region (Gouliermis et al. 2008).

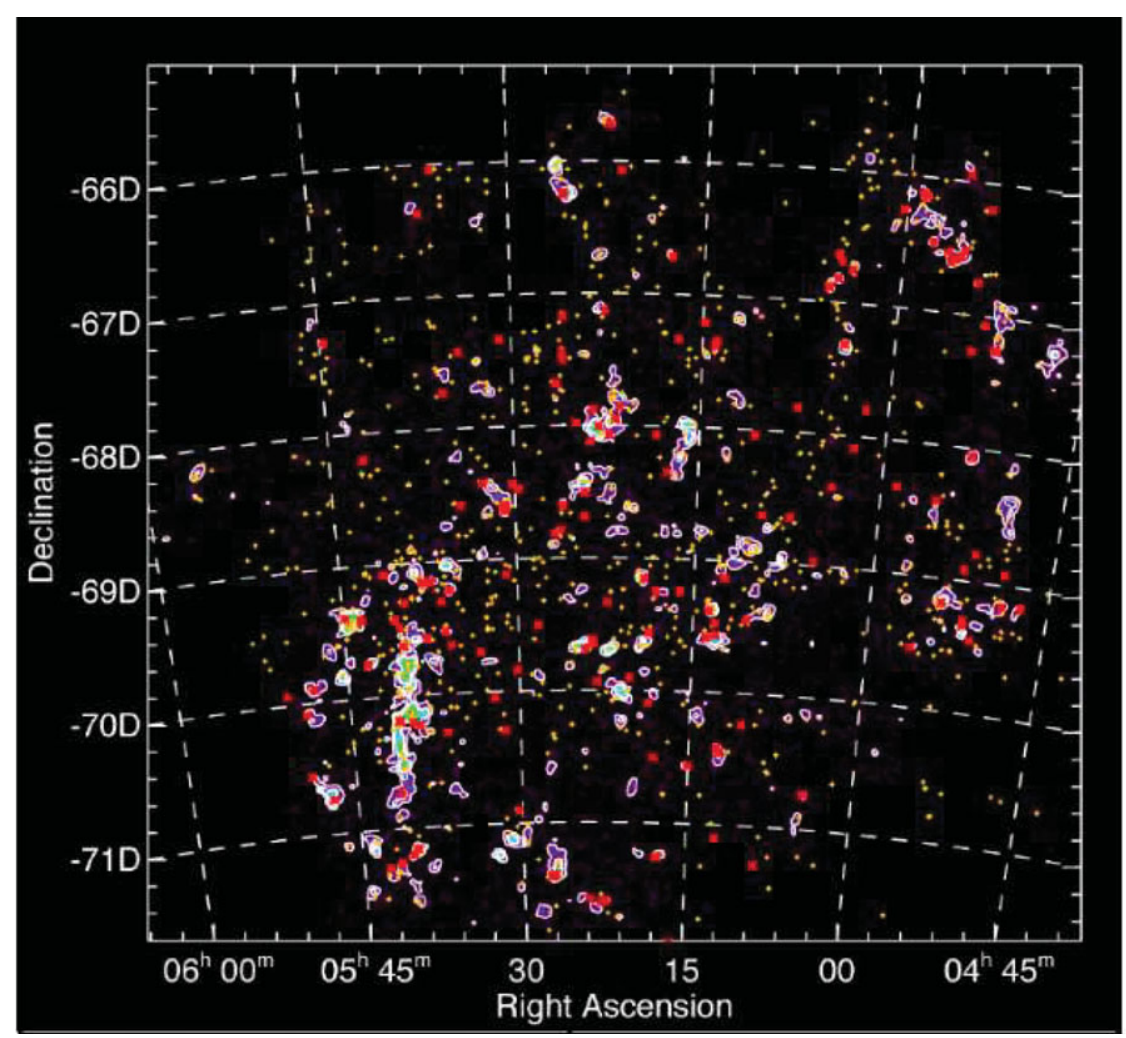

Figure 4. The NANTEN $\mathrm{CO}(1-0)$ map of the LMC. The distribution of the Spitzer YSO candidates (Whitney et al. 2008) are plotted as yellow crosses. The young OB association with ages less tha $10 \mathrm{Myr}$ are show in red dots. 


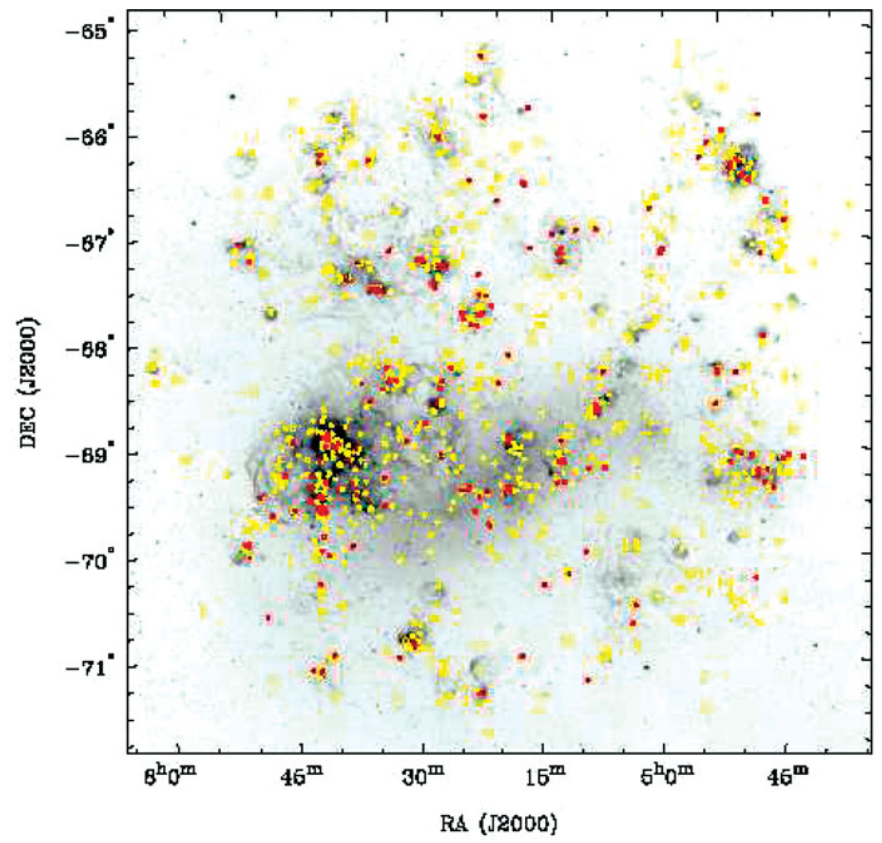

Figure 5. MCELS H $\alpha$ image of the LMC overlaid with positions of Spitzer YSO candidates (Chu, these proceedings). Sources shown in red have $\mathrm{M}_{8 \mu \mathrm{m}}<8$ mag and are possibly the most massive candidates while those in solid circles as considered as definitive YSOs following Gruendl et al. (2008).

In the LMC, Whitney et al. (2008), and Gruendl et al. (2008), have produced point sources catalogues from Spitzer IRAC images. The distribution of massive YSO candidates is well correlated to the molecular clouds as can be seen in Figure 4. A comparison between the Spitzer catalogues and the existing near-IR ground-based observations of massive YSOs in specific regions do not show a one-to-one correlation. This is probably due to the difficulty in recovering the point sources from the Spitzer images when these suffer fron strong contamination from the bright nebular emission in the region. Different selection criteria for analyzing the IRAC images and extracting point sources also contribute to this result. For example, Gruendl et al. (2008) has a YSO list for the LMC which differs from that of Whitney et al. (2008). We display their catalogue over an $\mathrm{H} \alpha$ image in Figure 5. In N11, Smith et al. (these proceedings) show more YSO candidates than the Whitney et al. list. In Figure 6, we show the distribution of the molecular gas and the YSOs as identified in the Whitney et al. catalogue for the N11 nebula and interestingly the well studied N11A and N11B regions where several near-IR embedded sources have been detected (Barbá et al. 2003) do not have Spitzer YSO counterparts. A similar situation is found in the massive star forming region, 30 Doradus, where the embedded YSO sources are not listed in the Spitzer point source catalogues.

The IRAC images have a resolution $6^{\prime \prime}$ at $8 \mu \mathrm{m}$ and thus cannot disentangle massive young stars which are born in compact groups. Spitzer colors of point sources can be contaminated by the other nearby stars and thus they may not show the typical YSO colors. Even for near-IR ground-based observations obtained with typical $0.6^{\prime \prime}$ seeing or better, 10 times better than the resolution of Spitzer images at $8 \mu \mathrm{m}$, multiple systems are not resolved. Only $H S T$ observations can resolve the multiple systems at the Magellanic 


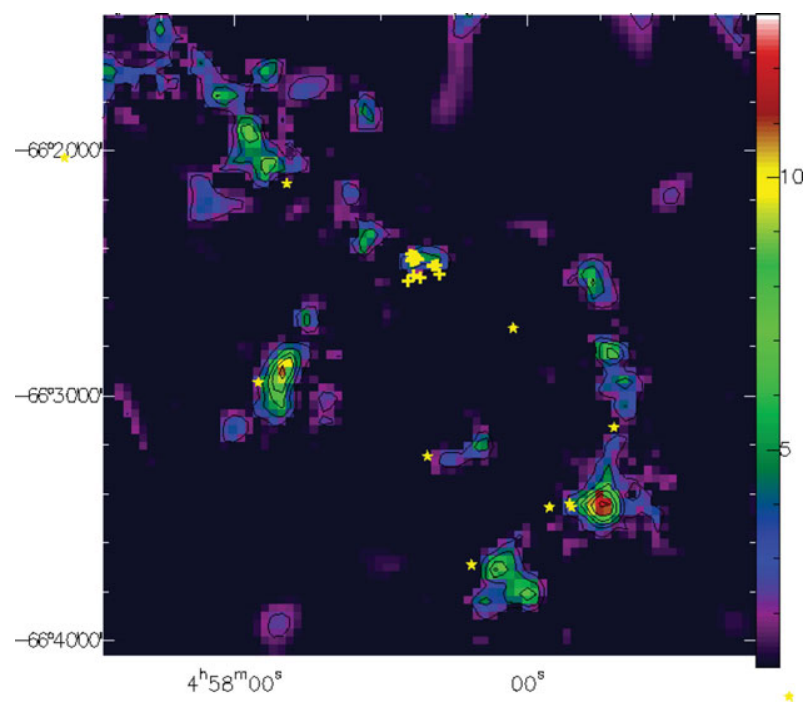

Figure 6. $\mathrm{CO}(1-0)$ image of $\mathrm{N} 11$. Overlaid are the positions of the YSO candidates (Whitney et al. 2008) as (yellow) stars. The N 11A and N 11B ground-based YSO positions (Barbá et al. 2003) are indicated as (yellow) crosses.

Clouds' distance. In 30 Doradus, the NICMOS/HST observations showed that several of the bright IR sources $\left(K_{\mathrm{S}} \sim 12\right)$ detected in ground-based photometry were compact multiple systems with 2 or 3 bright IR sources (Walborn et al. 1999), confirming that massive star formation occurs in compact groups. Thus, studies of these regions will require better algorithms to extract the point sources from the Spitzer images.

We know that massive star forming regions undergo several episodes of star formation. There is observational evidence of residual molecular clouds with dense clumps that could undergo new star formation. In 30 Doradus, N11, and N 66 a second generation of star formation is seen in the border of molecular clouds near the massive stars already formed. A particular case is that of N66 and its ionizing cluster, in which spectroscopically confirmed massive YSOs have been discovered located near the optical O stars, all of

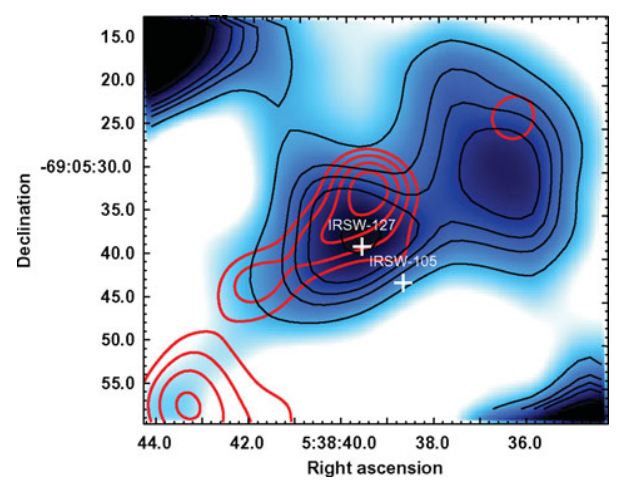

Figure 7. Dense molecular cloud at $20^{\prime \prime}$ from R 136 in 30 Doradus. The image shows the $\mathrm{CO}(2-1)$ integrated emission and superimposed in red are the $\mathrm{H}_{2} 2.12 \mu \mathrm{m}$ emission contours. Two crosses indicate the position of IRS W-105 and IRS W-127 (Rubio et al. 1998), embedded sources with typical YSO IR colors. 
them associated with a strong $\mathrm{H}_{2}$ molecular knot and $\mathrm{CO}$ peak. Thus, dense molecular clumps can survive the interaction of strong winds and high UV radiation field produced by the first generation of massive stars.

In a region with extreme physical ambient conditions such as 30 Doradus in the LMC, we have recently found a dense CO clump at only $20^{\prime \prime} \mathrm{NE}$ (i.e. a projected distance of $5 \mathrm{pc}$ ) of the ionizing compact cluster R 136 which contains $65 \mathrm{O}$ and $\mathrm{n}$ WR stars. This molecular cloud has a radius of $3 \mathrm{pc}$, a mass $\sim 1.5 \times 10^{4} \mathrm{M}_{\odot}$ and a mean density of $\sim 2.5 \times 10^{3} \mathrm{~cm}^{-3}$. The detection of $\mathrm{CS}(2-1)$ towards the CO peak indicates a density even higher, i.e. larger than $10^{6} \mathrm{~cm}^{-3}$. The molecular cloud, shown in Figure 7, is associated with an $\mathrm{H}_{2} 2.12 \mu \mathrm{m}$ emission knot and two IR sources with colors typical of a massive YSOs, probably embedded in the molecular cloud, are found (Rubio et al. 2008). This dense molecular cloud has survived the action of the strong winds and high UV radaition field of the massive stars in R 136.

Few studies have been done towards quiescent molecular clouds. In contrast to massive star forming regions these quiescent clouds show no sign of associated star formation. They were serendipitously discovered in $\mathrm{CO}(1-0)$ observations. The best studied is SMCB1\#1 (Rubio et al. 1993) which has a virial mass of $10^{4} \mathrm{M}_{\odot}$ and a gas mass as determined from continuum dust emission which is a factor of 10 larger ( $\mathrm{Ru}-$ bio et al. 2004). The cloud is illuminated externally by a B-type star (Reach et al. 2000) and the $24 \mu \mathrm{m}$ Spitzer image shows a larger spatial emission than the corresponding CO emitting volume. This cloud has been compared to have characteristics of a low-mass star forming regions such as $\rho$ Ophiuchus in our Galaxy (Boulanger, these proceedings).

\section{The Magellanic Bridge: a lower metallicity system}

The Magellanic Bridge has a metallicity even lower than the SMC, about 1/20 of the Galaxy. In such extreme ISM conditions, $\mathrm{CO}(1-0)$ emission was detected using the NANTEN telescope towards seven H I clouds with column densities exceeding $N(\mathrm{HI})>$ $10^{21}$ and bright $100 \mu \mathrm{m}$ emission, $I_{100 \mu \mathrm{m}}>2.6 \mathrm{MJy} \mathrm{sr}^{-1}$ (Mizuno et al. 2006). The properties of the $\mathrm{CO}$ clouds were determined from one single $\mathrm{CO}$ spectrum and these
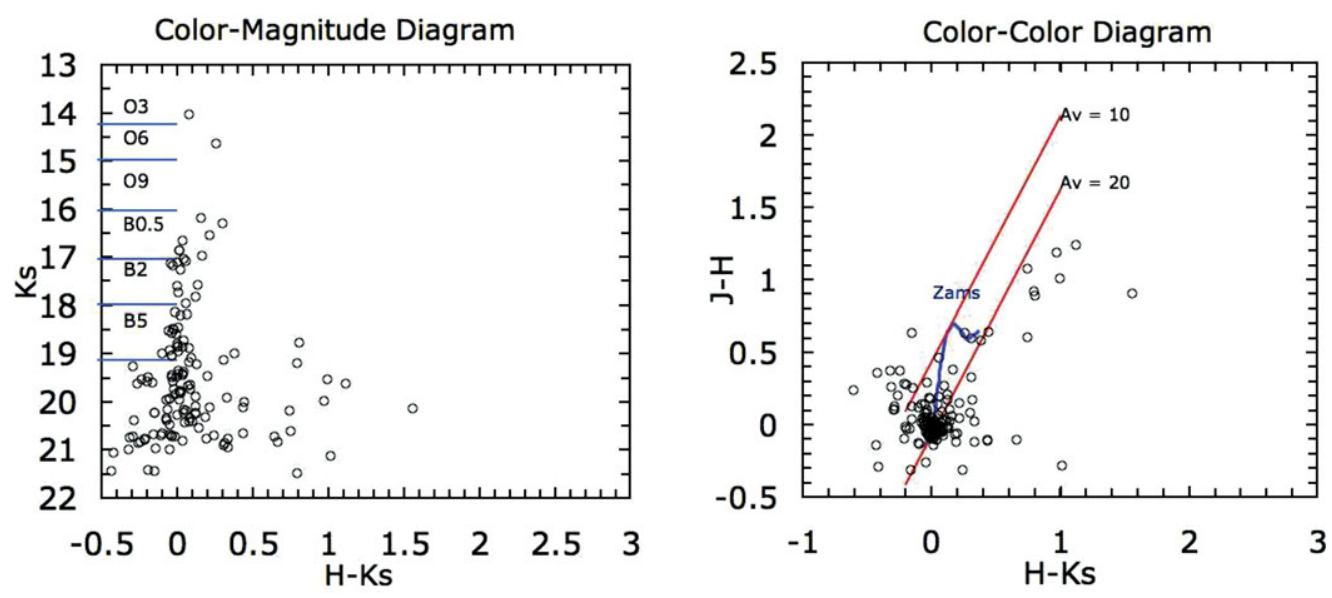

Figure 8. Color-Color and Color-Magnitude diagram of NGC 796 in the Magellanic Bridge molecular cloud. Several sources have color corresponding to Herbig Ae/Be stars while others are only seen in $K$. 
have CO luminosities of several $\sim 10^{3} \mathrm{~L}_{\odot}$ and their CO linewidths were in average smaller than the SMC molecular clouds CO linewidths and molecular masses of several $10^{3} \mathrm{M}_{\odot}$, determined from their CO lumninosities and using the NANTEN SMC conversion factor (Mizuno et al. 2001).

IR studies done by Nishiyama et al. (2007) towards the CO cloud associated to the NGC 796 cluster classified several IR sources as Herbig Ae/Be stars. We have done deep near-IR observations towards these $\mathrm{CO}$ clouds to investigate their embedded population using deep $J H K_{\mathrm{s}}$ imaging with the near-IR camera ISAAC on the VLT at Paranal Observatory last December 2007. Towards the molecular cloud associated with the cluster NGC 796, the IR color-color and color-magnitude diagrams, shown in Figure 8, show several IR sources that can be identified as Herbig Ae/Be stars or highly reddened O stars from their IR colors and position in the $J$ vs. $J-K$ diagram (Bik et al. 2006) Several of the brighter stars classified by Nishiyama et al. (2007) as Herbig Ae/Be stars are found to be multiple systems in our images. Several other weak sources are only seen in $K_{\mathrm{s}}$ ( $K \sim 18 \mathrm{mag}$ ) have been identified. (Rubio et al., these proceedings). A detailed study of this region is underway.

\section{Summary}

The Magellanic Clouds and in particular the SMC are ideal systems to study the process of star formation in a low metallicity ISM. Molecular clouds in these systems seem to be in virial equilibrium as they follow, in general, Larson's law. Nevertheless, there is evidence that SMC molecular clouds could be magnetically supported or in pressure bound equilibrium (Bot et al., these proceedings; Bolatto et al. 2008).

The molecular mass in these molecular clouds may not be traced by its $\mathrm{CO}$ emission as in spiral galaxies with Galactic metallicities and dust content. The CO molecule, used as standard tracer of the $\mathrm{H}_{2}$ is highly affected by the low dust content and the low $\mathrm{C}$ and $\mathrm{O}$ abundances permitting a high penetration of UV radiation into the interior of the molecular clouds and thus shielding of the CO molecule occurs at higher column densities of gas. In the SMC, where the metallicity and dust content are a factor of 10 lower than in the Milky Way, this column density is several $10^{22} \mathrm{~mol} \mathrm{~cm}^{-2}$.

The massive star forming regions in the Magellanic Clouds show a clear association to molecular clouds with masses $\sim 10^{4} \mathrm{M}_{\odot}$. These regions show on-going star formation and this is confirmed by a clear association between ground-based and Spitzer YSO candidates and the molecular clouds. Stars are formed in these metal-poor molecular clouds and also second generation of star formation occurs. Interestingly in regions of massive star formation with high UV radiation fiels and high winds residual dense molecular clumps are found in the neighbouring regions and these can undergo star formation. In many of the cases, the massive stars formed are found in compact clusters or multiple systems. Thus, the process of how stars form seems to be independent of the local conditons once a critical column density for the clouds is achieved.

The study of the IMF towards the molecular clouds in the Magellanic Clouds can be adressed with $8 \mathrm{~m}$ ground-based telescopes. At present, we are able to detect stars with masses as low as $1 \mathrm{M}_{\odot}$. Spitzer data could lower this limit but the spatial resolution is not adequate to resolve compact systems. The detailed study of the dense molecular cores at the LMC and SMC will be possible when ALMA starts operation.

\section{Acknowledgements}

M.R wishes to acknowledge support from FONDECYT (CHILE) Grant No 12345608. She is supported by the Chilean Center for Astrophysics FONDAP No. 15010003. 


\section{References}

Barbá, R. H., Rubio, M., Roth, M, et al. 2003, AJ, 125, 1940

Bik, A., Kaper, L., Waters, L. B. F. M., et al. 2006, A\& $A$, 455, 561

Bloemen, J. B. G. M., Strong, A. W., Mayer-Hasselwander, H. A., et al. 1986, A\& A, 154, 25

Bolatto, A. D., Simon, J. D., Stanimirović, S., et al. 2007, ApJ, 655, 212

Bolatto, A, Leroy, A., Rosolowsky, E., et al. 2008, ApJ, 686, 948

Bot, C., Boulanger, F., Lagache, G., Cambrésy, L., \& Egret, D. 2004, A\& $A, 423,567$

Bot, C, Boulanger, F., Rubio, M., \& Rantakyrö, F. 2006, A\&A, 471, 103

Brandner, W., Grebel, E., Barbá, R., et al. 2001, AJ, 122, 858

Cohen, R. S, Dame, T. M, Garay., G., et al. 1988, ApJ, 331, L95

Contursi, A., Lequeux, J., Cesarsky, D., et al. 2000, A\&A, 362, 310

Contursi, A, Rubio, M., Sauvage, M., et al. 2007, A\&SA, 469, 539

Davies, R. D., Elliot, K. H., \& Meaburn, J. 1976, MemRAS, 81, 89

Dupac, X., del Burgo, C., Bernard, J.-P., et al. 2003, MNRAS, 344, 105

Fukui, Y, Mizuno, N., Yamaguchi, R., et al. 1999, PASJ, 51, 745

Fukui, Y, Kawamura, A., Minamidani, T., et al. 2008, ApJS, 178, 56

Galliano, F., Madden, S. C., Jones, A. P., Wilson, C. D., Bernard, J. -P., \& Le Peintre, F. 2003, $A \mathscr{E} A, 407,159$

Galliano, F., Madden, S. C., Jones, A. P., Wilson, C. D., \& Bernard, J. -P. 2005, A\& A, 434, 867

Gouliermis, D., Brandner, W., \& Henning, Th. 2006, ApJ, 636, L133

Gouliermis, D. A., Chu, Y. -H., Henning, Th., et al. 2008, ApJ, arXiv:0710.1352

Gruendl, R. A., Chu, Y., et al. 2008, submitted

Henize, K. H. 1956, ApJS, 2, 315

Israel, F. P., Johansson, L. E. B., Lequeux, J., et al. 1993, A\& A, 276, 25

Jones, T. J., Woodward, C. E., Boyer, M. L., et al. 2005, ApJ, 620, 731

Larson, R. B. 1981, MNRAS, 194, 809

Lequeux, J., Le Bourlot, J., Pineau des Forêts, G., Roueff, E., Boulanger, F., \& Rubio, M. 1994, $A \mathscr{E} A, 292,371$

Leroy, A., Bolatto, A., Stanimirović, S., Mizuno, N., Israel, F., \& Bot, C. 2007, ApJ, 658, 1027

Massey, P. 2002, ApJS, 141, 81

Meixner, M., Gordon, K., Indebetouw, R., et al. 2006, AJ, 133, 2268

Mizuno, N, Rubio, M., Mizuno, A., Yamaguchi, R., Onishi, T., \& Fukui, Y. 2001, PASJ, 53, 971

Mizuno, N., Muller, E., Maeda, H., et al. 2006, ApJ, 643, 107

Nishiyama, S., Haba, Y., Kato, D., et al. 2007, ApJ, 658, 358

Nota, A., Sirianni, M., Sabbi, E., et al. 2006, ApJ, 640, L29

Oliveira, J. M., van Loon, J. Th., Stanimirović, S., \& Zijlstra, A.A. 2006, MNRAS, 373, 75

Perez, L. 2005, MSc thesis, Universidad de Chile, Santiago, Chile

Reach, W. T., Boulanger, F., Contursi, A, et al. 2000, A\&A, 361, 895

Rubio, M., Garay, G, Montani, J., et al. 1991, ApJ, 368, 173

Rubio, M., Lequeux, J., Boulanger, F., et al. 1993, A\&\&A, 271, 1

Rubio, M., Barbá, R. H., Walborn, N., et al. 1998, AJ 116, 1708

Rubio, M., Contursi, A., Lequeux, J., et al. 2000, A\&A, 359, 1139

Rubio, M., Boulanger, F., Rantakyrö, F., \& Contursi, A., 2004, A\&A, 425, L1

Rubio, M. 2007, in B. G. Elmegreen \& J. Palouš (eds.), Triggered star formation in a turbulent ISM, IAU Conf.Proc. 237 (Cambridge: CUP), p. 40

Rubio, M., \& Barbá, R. H. 2008, ApJL, submitted

Rubio, M., Paron, S., \& Dubner, G. 2008, A\&AA, submitted

Sabbi, E., Siriani, M, Nota, A., et al. 2008, AJ, 135, 173

Simon, J. D., Bolatto, A. D., Whitney, B. A., et al. 2007, ApJ, 669, 327

Solomon, P. M., Rivolo, A. R., Barrett, J., \& Yahil, A. 1987, ApJ, 319, 730

Walborn, N. R., Barbá, R. H., Brandner, W., et al. 1999, AJ, 117, 225

Weingartner, J. C., \& Draine, B. T. 2001, ApJ, 548, 296

Whitney, B. A., Sewilo, M., Indebetouw, R., et al. 2008, AJ, 136, 18

Yamaguchi, R., Mizuno, N., Mizuno, A., et al. 2001, PASJ, 53, 985 